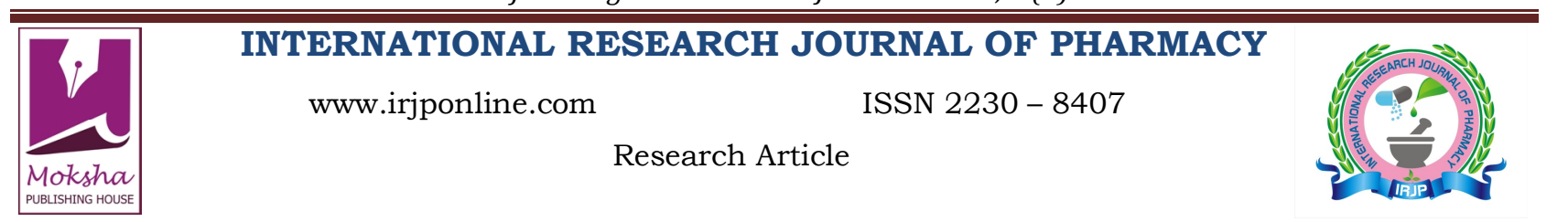

\title{
EVALUATION OF IN VITRO STABILITY STUDIES ON NUTRACEUTICALS IN ORAL SOLID DOSAGE FORMS WITH SPECIAL REFERENCE TO GLUCOSAMINE
}

Raju Merugu ${ }^{1 *}$, M. Vijaya Bhaskara Reddy ${ }^{2}$ and Rajendra G. Lala ${ }^{3}$

${ }^{1}$ Research Scholar, Department of Pharmacy, Vinayaka Mission's Research Foundation Deemed University, Tamil Nadu, India

${ }^{2}$ Department of Biotechnology, Vinayaka Mission's Research Foundation Deemed University, Tamil Nadu, India

${ }^{3}$ Research Supervisor, Department of Pharmacy, Vinayaka Mission's Research Foundation Deemed University, Tamil Nadu, India

*Corresponding Author Email: meruguraju@gmail.com

Article Received on: 10/08/13 Revised on: 21/08/13 Approved for publication: 22/08/13

DOI: $10.7897 / 2230-8407.04854$

IRJP is an official publication of Moksha Publishing House. Website: www.mokshaph.com

(C) All rights reserved.

\section{ABSTRACT}

Pure Glucosamine is very "hygroscopic" and degrades (breaks down) rapidly when exposed to moisture or air. To avoid this, Glucosamine needs to be bound to a stabilizer to be sold commercially. The sulfate and the HCL forms are two of the most common "agents" that Glucosamine is bound to ensure its stability. After Glucosamine is bound, it is stable and will not degrade before it can get to the store shelf. Hence it is very difficult to prepare Glucosamine base and instead find Glucosamine Sulfate or Glucosamine HCL. Thus an attempt was made to stabilize the Glucosamine formulation by using the combination of antioxidant, Desiccant to resolve the problems associated with the Glucosamine formulation. In the present formulation studies were carried for the stability studies as ICH guidelines with Real time and Accelerated stability studies and it was found to be stable during the study period of stability for three months. All the formulas with single desiccant and single ant-oxidant did not reduce the extent of the degradation. The tablets so prepared with conventional methods showed good results physical evaluation parameters and chemical parameters such as Assay and Dissolution values. The granules prepared by using these antoxidants and desiccant, were good in their flow properties. Glucosamine, an amino monosaccharides naturally occurring in the connective and cartilage tissues, contributes to maintaining the strength, flexibility and elasticity of these tissues. Glucosamine is a precursor to a molecule called a glycosaminoglycan, which is used in the formation and repair of cartilage. In recent years, glucosamine has been used widely to treat osteoarthritis in humans and animal models. In vivo, glucosamine is typically converted to $\mathrm{N}$-acetyl-glucosamine. Non-steroidal anti-inflammatory drugs (NSAIDS) are effective in reducing inflammation but are not highly soluble and, in addition, may have undesirable side effects. Efforts have been made to improve the pharmaceutical properties of NSAIDs, such as permeability, solubility and stability, by creating NSAID prodrugs. Prodrugs are typically evaluated in relation to the drug's pharmacological and pharmacokinetic properties. These modifications may alter the physicochemical properties of the drug, which may in turn effect the administration options that optimize drug delivery.

Keywords: Nutraceuticals, in-vitro, stability study, glucosamine

\section{INTRODUCTION}

In present scenario, rapid industrialization and technological advancement has led to improved quality of life in terms of income, spending and lifestyle along with the economic growth. The first victim of this lifestyle change has been food habits. It has also imposed a major challenge in the form of 'lifestyle diseases'. Consumption of junk food has increased enormously, which has led to a number of diseases related to nutritional deficiencies. Nutraceuticals can play an important role in controlling them. The idea behind the mode of action of nutraceuticals is to provide functional benefits by increasing the supply of natural building blocks in the body. Replacement of these building blocks can work in two ways: to diminish disease signs or to improve performance. Nutraceuticals is a broad umbrella term used to describe any product derived from food sources that provides extra health benefits in addition to the basic nutritional value found in foods. Nowadays, Nutraceuticals are available in various forms including tablets. The formulations are a combination of natural ingredients, essential vitamins, amino acids and minerals, offer health benefits far beyond vitamin supplements alone. Oral route has been one of the most popular routes of drug delivery due to its ease of administration, patient compliance, least sterility constraints and flexible design of dosage forms. In the last few years controlled release dosage forms have made significant progress in terms of clinical efficacy and patient compliance. The objective of designing a controlled release system is to deliver drugs at a rate necessary to achieve and maintain a constant drug slowly over several hours, to protect the drug the stomach from the irritating effects of the drug. In present scenario, rapid industrialization and technological advancement has led to improved quality of life in terms of income, spending and lifestyle along with the economic growth. The first victim of this lifestyle change has been food habits. It has also imposed a major challenge in the form of 'lifestyle diseases'. Consumption of junk food has increased enormously, which has led to a number of diseases related to nutritional deficiencies. Nutraceuticals can play an important role in controlling them. The idea behind the mode of action of nutraceuticals is to provide functional benefits by increasing the supply of natural building blocks in the body. Replacement of these building blocks can work in two ways: to diminish disease signs or to improve performance. Nutraceuticals is a broad umbrella term used to describe any product derived from food sources that provides extra health benefits in addition to the basic nutritional value found in foods ${ }^{1}$. Controlled drug delivery system is one which delivers the drug at a predetermined rate locally or systemically for a period of time i.e. it implies a predictability and reproducibility in the drug release kinetics which means that release of active ingredient from a controlled release drug delivery system proceeds at a rate profile that is not only predictable kinetically but also reproducible from one unit to other. Polymer structure features contributing to mucoadhesion $^{2}$ Leung S .S et.al. Studied, the expanded nature of mucin and polymer networks; which permits mutual interpenetration / Inter diffusion of mucin and adhesives results in an increase in contact area and establishment of physical entanglement of two different macromolecules. In 
vivo and In vitro nasal mucoadhesion of some water-soluble polymer: A cationic polymer with higher charge density causes the stronger adhesion of aqueous solution on the mucosa. This fact suggests that electronic interaction between the polymer base and mucosa may be factor for adhesion ${ }^{3}$. Studies on the drug release kinetics from carbomer matrices: Durrani M.J et.al showed that, the drug solubility can influence the mechanism of drug release. Atenolol is a sparingly water-soluble drug demonstrated a square root time dependent drug release. While Furosemide, poorly watersoluble drug gave zero-order release. So the solubility is having the influence on the drug release in controlled release tablet ${ }^{4}$. It is totally independent on the various grade of the polymer. Gastro-Retentivity: Its drug delivery potential: ${ }^{5}$ Singh $\mathrm{M}$ et.al showed that, that the extent of drug absorption from GIT is determined by GI Physiology, irrespective of controlled release properties of the device. These include gastro retentive system, delayed release system and colon targeting. Various other approaches have been tried to retain the dosage form in the stomach as a way of increasing the overall rotation time and include floating system, high density pallets, bioadhesive system, swelling systems and shape system. Uses of Passage dealing precipitants have recently been highlighted. E.g. Salts of Myrestic acid. Improvement of drug release rate from carbopol 934P formulation: Nakanishi T, Kaiho $\mathrm{F}$ and Hayashi $\mathrm{M}$ having finding that, the carboxyl group of Carbopol is dissociate in the alkaline environment, electrostatic repulsion between negatively charged carboxyl groups causes uncoiling and expansion of molecules resulting in swelling of the polymer and gel formation. The gel is composed of closely packed swollen particles, whose swelling increases with increase in the $\mathrm{pH}$; thus forming thicker and more rigid gel layer ${ }^{6}$. Aminophylline bio adhesive tablets attempted by wet granulation $^{7}$ the wet granulation has limited bio adhesion, because wetting of Carbomer and there drying was impossible when their concentration exceed $10 \%$. Wet granulation did not allow obtaining of highly bio adhesive tablets. However wetting and drying steps did not alter the polymer structure or bio adhesive property. Factors affecting the bio adhesive properties of tablets consisting of Hydroxy propyl cellulose Carboxyvinyl polymer: ${ }^{8}$, Saoh K et.al having findings which shown that, the adhesion force was closely related to the moisture content on the mucus membrane. Namely the tablet did not stick to a very moist membrane, but stick tightly to one with little moisture. Hosseinali Tabandeh, et al. has designed the sustained release matrix tablet of Aspirin with Ethylcellulose, Eudragit RS100 and Eudragit S100 and studied the release profile, tablet hardness of the tablets ${ }^{9}$. Glucosamine $\mathrm{HCl}$ was found to be effective in controlling osteoarthritis (OA) symptoms in several clinical studies. In particular, two randomised, placebo-controlled, double-blind trials of 3-year duration in knee OA patients, showed that this symptom-modifying effect is sustained over long-term treatment courses. Moreover, both studies indicated that the drug also has a structure-modifying effect, as assessed by measurement of joint space narrowing on standardised plain radiographs by a valid technique and consistent within studies and patient populations. ${ }^{10}$

\section{MATERIALS AND METHODS}

Glucosamine Hydrochloride (Yantai Dongcheng Biochemicals Co. Ltd), Dicalcium Phosphate, Anhydrous (Rhodia), Carbomer (Lubrizol), Stearic acid (Taurus chemicals limited) Ammonio Methacrylate Copolymer Type`
A and B (Eudragit Rohm Pharma Polymers / Degussa), Purified Talc (Luzenac), Ethanol (Ethyl alcohol-SD Fine Chemicals), IPA, Citric acid, Sodium starch glycollate, Tartaric acid, HPC, Colloidal silicon dioxide, PVPK 30, Ethyl cellulose, Crospovidone, Sodium metabisulphate, Mannitol, PEG 600, EDTA and Kieselgur. Analytical Method development, Drug Product specification, Description, Identification, Average mass, Uniformity of mass, Dimension, Hardness, Friability, Assay and dissolution.

\section{Drug Release Profiles}

$1^{\text {st }}$ hour ------between $20 \%$ to $30 \%$ of label claim

$4^{\text {th }}$ hour ----- between $40 \%$ to $55 \%$ of label claim

$8^{\text {th }}$ hour------ between $60 \%$ to $75 \%$ of label claim

$12^{\text {th }}$ hour-----Not Less Than $85 \%$ of label claim

Dissolution conditions: Apparatus: USP Type II (Paddle)

Medium

Volume

RPM

Time intervals

Temperature

$$
\begin{aligned}
& \text { Distilled water. } \\
& 1000 \mathrm{~mL} \text {. } \\
& 50 . \\
& 1^{\text {st }}, 4^{\text {th }} 8^{\text {th }} \text { and } 12^{\text {th }} \text { hour. } \\
& 37,0^{\circ} \mathrm{C} \pm 0,5^{\circ} \mathrm{C} \text {. }
\end{aligned}
$$

\section{Chromatographic Conditions}

Column : BDS Hypersil $\mathrm{C}_{8} ; 250 \mathrm{~mm} \mathrm{x} \mathrm{4,6} \mathrm{mm;} 5$

$\mu \mathrm{m}$ or equivalent.

Flow rate $\quad: 0,6 \mathrm{~mL} /$ minute

Detector : UV at $195 \mathrm{~nm}$

Injection volume: $10 \mu 1$.

Temperature : Ambient.

Buffer: Mix 1, $0 \mathrm{~mL}$ of Phosphoric acid in $2000 \mathrm{~mL}$ of distilled water, adjust to $\mathrm{pH}$ 3, 0 with Potassium Hydroxide.

Mobile Phase: Mix $900 \mathrm{~mL}$ of Buffer and $100 \mathrm{~mL}$ of Acetonitrile. Sonicate for 15 minutes and filter through 0, 45 $\mu$ membrane filters and degas.

\section{Manufacturing Procedure of B2, B3, B4, B5, B6, B7 and B8}

Step 1: Sift item numbers 1, 2, 3 and 4 through \#40 ASTM S.S. sieves and collected separately into polyethylene bags.

Step 2: Blended item 1, 2 and 3 of step 2 in a separate polybag and mixed for five minutes

Step 3: Load dry mix of step 2 into RMG and add Isopropyl alcohol at slow speed of impeller and Mix for 5 minutes (until to get a breakable dough mass)

Step 4: Load the wet mass into Rapid dryer, dry at $40^{\circ} \mathrm{C}$ for 15 minutes and to get LOD NMT $2 \% \mathrm{w} / \mathrm{w}$

Step 5: Dried granule pass through \#20 and collected in poly bag.

Step 6: Blended step 5 and item no 4 for 2 minutes in polybag.

Step 7: Lubricated blend compressed by 23.5 X $11.50 \mathrm{~mm}$ punches at the average weight of $1875 \mathrm{mg}$.

\section{Preparation of Coating Suspension}

Step 8: Dissolve to disperse Ammonio Methacrylate Copolymer Type A and B in alcohol and Add purified talc, to getting homogenous suspension free from lumps.

Step 9: Loaded uncoated tablets into conventional coating pan.

Step 10: Applied coating suspension on uncoated tablets to desired weight gain achieved.

The physical and chemical parameters of the tablets were found to be satisfactory. 
Stability Study

Stability study was carried out on the formulation B5. Tablet of batch B5 were packed in PVC blister and180 cc HDPE container with silica gel bag and absorbent cotton. It was stored at $40 \pm 2^{\circ} \mathrm{C}, 75 \% \pm 5 \%$ relative humidity for $1,2,3$ and 6 months. Tablets were evaluated for in-vitro drug release after six month. Result obtained was compared with data obtained for zero ambient time at ambient temperature. The results of in-vitro release study of Batch B5 after stability study.

\section{In vitro Release Study}

Glucosamine sulphate release was determined with six tablets per formulation using a USP Type II dissolution assembly
(Hansen SR-8 Plus, Chatsworth, CA, and Erweka DT-70, Milford, CT) in deionized water with a paddle speed of 50 $\mathrm{rpm}$ and bath temperature of $37.0+0.5^{\circ} \mathrm{C}$. Samples were collected interval of 15 minutes, 30 minutes and 45 minutes. Each samples were collected were replaced with fresh equal volume of dissolution media. Six replicates were tested for each batch of the tablet formulations. Dissolution samples were filtered and this apparatus was equipped for filtered fraction collection diluted to appropriate concentrations for drug analysis. Each filtered sample was analyzed by HPLC method using Waters Alliance 2695 Seperations module; 2996 PDA detector was used with BDS Hypersil C8 column (250 $\mathrm{mm}$ x $4.6 \mathrm{~mm} ; 5 \mu \mathrm{m}$ particle sizes)

Table 1: Shows the Data of Glucosamine Stability Study and in-vitro Release Study of Batch B5 for 6M

\begin{tabular}{|c|c|c|c|c|c|}
\hline & \multicolumn{5}{|c|}{ \% Cumulative drug release profiles } \\
\hline Time in hour & Spec & Initial & $\mathbf{1 M}$ & $\mathbf{3 M}$ & $\mathbf{6 M}$ \\
\hline 0 & 0 & 0 & 0 & 0 & 0 \\
\hline 1 & 30 & 28 & 30 & 32 & 30 \\
\hline 4 & 55 & 52 & 52 & 54 & 55 \\
\hline 8 & 75 & 72 & 72 & 75 & 72 \\
\hline 12 & 85 & 92 & 95 & 93 & 92 \\
\hline
\end{tabular}

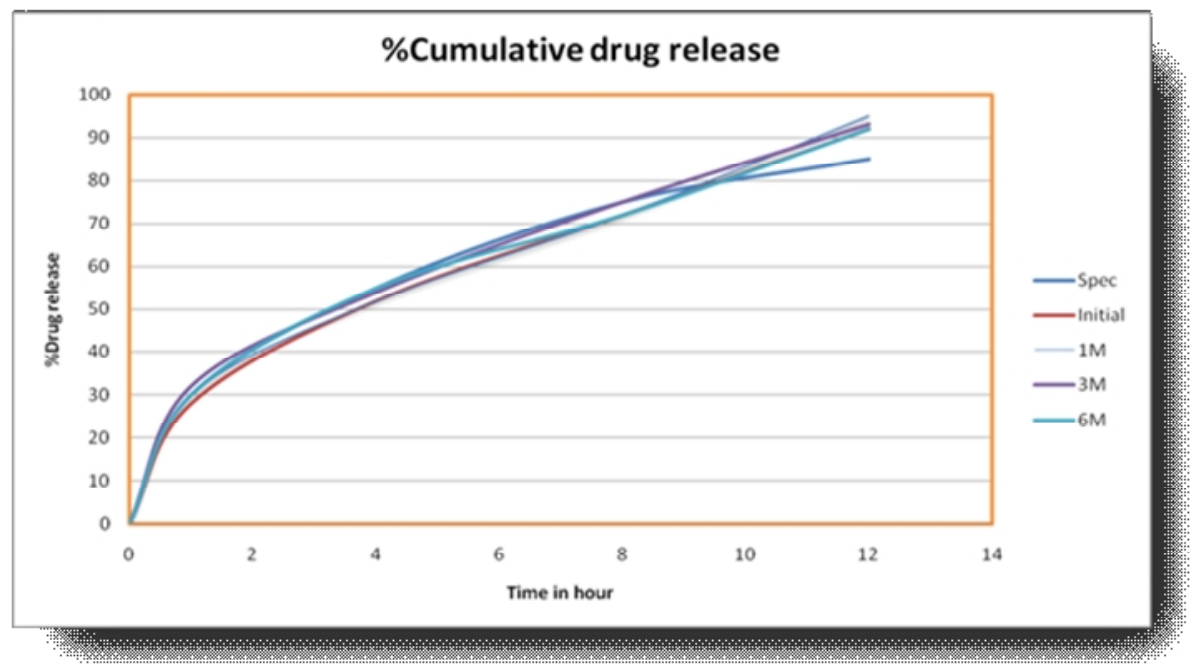

Figure 1: Dissolution Profile of Batch B5 for 6M Stability Data

The Batch B5 did not show any physical or chemical interaction between drug and polymer. It was concluded from the IR Study showed similar peak of drug in tablet formulation.

\section{RESULTS AND DISCUSSION}

In the present study the results shows various formulations of sustained release tablets of Glucosamine Hydrochloride were developed using various polymers viz, Carbomer and Ammonio Methacrylate Copolymer Type A and B different proportions and combinations by wet granulation technique. The tablets were evaluated for physical characterization, in vitro release study and stability studies (Figure 1 and Table 1). Results of in vitro release profile indicated that formulation (B5) was the most promising formulation as the extent of drug release from this formulation was high as compare to other formulations, which are suitable for once a day medication (Figure 1 and Table 1). Stability study was conducted on tablets of Batch B5 at $40 \pm 2{ }^{\circ} \mathrm{C}, 75 \pm 5 \% \mathrm{RH}$ for upto 6 months. Tablets were evaluated for in vitro release profile, after six month. No significant changes were observed in any of the studied parameters during the study period, thus it could be concluded that formulation was stable (Figure 1 and Table 1). The synthesis and initial physicochemical evaluation of model mutual prodrugs as potential osteoarthritis therapeutics show some theoretical promise. Similar applications are of the pharmaceutical industry's interest. One objective was met with the synthesis of NSAID-glucosamine mutual prodrugs. These models may or may not improve the pharmaceutical properties of NSAIDs and glucosamine such as their permeability, solubility and stability in relationship to each drug's pharmacological and pharmacokinetic properties. The modifications have altered the physiochemical properties that will affect delivery methods by optimizing the drug's delivery, pharmacodynamic and pharmacokinetic properties. A drawback to the use of a concomitant (mutual prodrug) application of a product delivering an NSAID and glucosamine is that it currently would immediately raise 
questions by FDA, since glucosamine is not considered a drug and its efficacy has not been proven. Hence, further in vitro and especially in vivo studies must be undertaken. Therefore, if these products or similar products show clinical significance a new drug application (NDA) would have to be filed due to the claims needed to protect the glucosamine entity. Predictive science inherently says that, like the "statindrugs", that were developed from nutraceuticals, glucosamine based products will be developed into "blockbuster" drugs with proven efficacies under FDA protocol. From the present investigation it is concluded that the tablets of batch B5 had considerable in vitro drug release. Tablets of Batch B5 was selected as an optimum batch and evaluated for further parameter like accelerated stability study and characterization using IR spectroscopy. The stability study revealed that there was not significant change in dissolution profile for a period of 6 months. Tablet of batch B5 did not show any physical or chemical interaction between drug and polymer, which was concluded from the IR Study showed similar peak of drug in tablet formulation. These modifications may alter the physicochemical properties of the drug, which may in turn effect the administration options that optimize drug delivery.

\section{REFERENCES}

1. MD. HJH. Nutripharmaceuticals for osteoarthritis best practice and research clinical rheumatology 2001; 15(4): 595-605.

2. Leung SS, Robinson JR. Polymer structure features contributing to mucoadhesion. II Journal of controlled release 1990; 12: 178-194. http:// dx.doi.org/10.1016/0168-3659(90)90099-F
3. Bell CL and Peppas NA. An apparatus to measure polymer swelling under load; Int. Journal of pharmaceutics 1996; 134(1-2): 167-172.

4. Durrani MJ, Andrews A, Whitaker R and Benner SC. Studies on drug release kinetics from carbomer matrices; Drug. Dev. Ind. Pharmacy 1994;20(15):2439-2447. http://dx.doi.org/10.3109/03639049409042648

5. Singh M. Gastro-retentivity: its drug delivery potential; Indian journal of pharmaceutical sciences 2002; may-june: 1-2.

6. Nakanishi T, Kaiho F and Hayashi M. Improvement of drug release rate from Carbopol 934P formulation; Chem. Pharm. Bull 1998; 46(1): 171173. http://dx.doi.org/10.1248/cpb.46.171

7. Prudat Christianes $\mathrm{C}$, Arnaud $\mathrm{P}$, Allain $\mathrm{P}$ and Chaumeil JC. Aminophylline bio adhesive tablets attempted by wet granulation; Int. Journal of pharmaceutics 1996; 141: 109-116. http://dx.doi.org $/ 10.1016 / 0378-5173(96) 04622-4$

8. Saoh K, Takayama K, Machida Y, Suzuki Y, Nakagaki M and Naga T. Factors affecting the bio adhesive property of tablets consisting of Hydroxypropyl cellulose Carboxyvinyl polymer; Chem. Pharm. Bull 1989; 37(5): 1366-1368. http://dx.doi.org/10.1248/cpb.37.1366

9. Hosseinali Tabandeh, Seyed Alireza Mortazavi, Tina Bassir Guilani. Preparation of sustained release matrix tablet of aspirin with Ethylcellulose, Eudragit RS100 and Eudragit S100 and studying the release profiles and their sensitivity to tablet hardness. Iranian journal of pharmaceutical Journal 2003; 2: 201-206.

10. Persiani S. Glucosamine oral bioavailability and plasma pharmacokinetics after increasing doses of crystalline glucosamine sulphate in man. Osteoarthritis and Cartilage 2005; 13: 1041-1049. http://dx.doi.org/10.1016/j.joca.2005.07.009 PMid:16168682

Cite this article as:

Raju Merugu, M. Vijaya Bhaskara Reddy and Rajendra G. Lala. evaluation of in vitro stability studies on nutraceuticals in oral solid dosage forms with special reference to Glucosamine. Int. Res. J. Pharm. 2013; 4(8):265-268 http://dx.doi.org/10.7897/2230-8407.04854 